\title{
Effect of the size of the charged group on the properties of alkoxylated NFCs
}

\author{
Ali Naderi · Per Tomas Larsson · Jasna S. Stevanic • Tom Lindström • Johan Erlandsson
}

Received: 15 October 2016/Accepted: 1 January 2017/Published online: 9 January 2017

(C) The Author(s) 2017. This article is published with open access at Springerlink.com

\begin{abstract}
The impact of the size of the charged group on the properties of alkoxylated NFC was studied by two chloroalkyl acid reagents. It was found that the employment of the larger 2-chloropropionic acid reagent leads to improved properties, e.g. higher fraction of nano-sized materials, and significantly better redispersion as compared to when the smaller monochloroacetic acid was employed. The differences in the impacts of the different reagents were hypothesized to be due to a more efficient disruption of the cohesion between the nanofibrils when a larger charged group was employed.
\end{abstract}

Keywords Nanofibrillated cellulose (NFC) . Alkoxylation - Redispersion - Degree of fibrillation . Barrier properties $\cdot$ NMR

A. Naderi $(\bowtie) \cdot$ P. T. Larsson · J. S. Stevanic .

T. Lindström

Innventia AB, Drottning Kristinasv 61, 11486 Stockholm,

Sweden

e-mail: ali.naderi@innventia.com

\section{J. Erlandsson}

Department of Fibre and Polymer Technology, KTH -

Royal Institute of Technology, Teknikringen 56,

10044 Stockholm, Sweden

\section{Introduction}

Nanofibrillated cellulose (NFC) is a material with several exciting properties, and is therefore potentially of interest for numerous applications (Dufresne 2013; Lindström et al. 2014). Today, NFC is used in the pulp and paper industry to strengthen ${ }^{1}$ paper and cardboard products, is applied in e.g. cosmetics $^{2}$ as a rheological modifier and is used as an odour-eliminating agent in diapers. ${ }^{3}$ A broader employment of NFC requires, however, the overcoming of several challenges. For example, the production of transparent NFC-films (Siró et al. 2011) and strong NFC-based filaments (Håkansson et al. 2014) requires NFCs with low fibre fragment content. In addition, several applications require concentrated or completely dried NFC products that can be redispersed and diluted to a desired consistency. The concentrated NFC should therefore be redispersible, that is to possess the ability to regain its original properties when redispersed and diluted by industrially relevant processes-this constitutes a significant challenge (Naderi et al. 2015b).

\footnotetext{
$\overline{1}$ http://www.paperage.com/2011news/05_31_2011stora_enso_ imatra.html.

${ }^{2}$ http://www.pulpapernews.com/2015/09/oji-to-develop-nano cellulose-for-cosmetic-applications.

${ }^{3}$ http://www.japantimes.co.jp/news/2015/11/27/business/corpo rate-business/nippon-paper-invest-nanofibre-head-off-challengesprint/.
} 
It is known that the fibre fragment-contents of NFCs are mainly determined by the chemical pretreatment process during NFC production (Naderi et al. 2016b). It is also known that the pre-treatment processes that enable a high degree of fibrillation (low fibre fragment content) are those that can chemically ${ }^{4}$ attach a significant amount of charges onto the nanofibrils (Wågberg et al. 1987; Isogai et al. 2011; Tejado et al. 2012; Yano et al. 2011; Noguchi et al. 2014). In this context, it has been noted that NFCs with higher charge densities have shown better redispersibility (in water) compared to the lower charged equivalents (Eyholzer et al. 2010; Naderi et al. 2015b). However, the positive effects that are obtained by increasing the charge density are offset by a lowered dewatering speed of NFC, which hampers many potential applications. Hence, strategies must be developed to improve the redispersibility and degree of fibrillation of NFCs, at as low charge densities as possible.

In this report, the impact of the size of the charged groups on the degree of fibrillation and the redispersibility of alkoxylated NFCs was investigated. The hypothesis was that increasing the size of the charged group leads to a more effective disruption of the cohesive forces between the nanofibrils, enabling the production of NFCs with a high degree of fibrillation and attractive redispersion properties.

\section{Materials and methods}

\section{Materials}

A commercial never-dried TCF-bleached sulfite dissolving pulp (trade name: Dissolving Plus) from a mixture of Norway spruce $(60 \%)$ and Scottish pine (40\%) was obtained from Domsjö Fabriker (Domsjö Mill, Sweden). The pulp had a hemicellulose content of $4.5 \%(\mathrm{w} / \mathrm{w})$ (measured as solubility in $18 \% \mathrm{NaOH}$, $\mathrm{R} 18)$ and a lignin content of $0.6 \%(\mathrm{w} / \mathrm{w})$.

Ethanol was purchased from VWR (Sweden). Monochloroacetic acid $\left(\mathrm{ClCH}_{2} \mathrm{CO}_{2} \mathrm{H}\right)$, 2-chloropropionic acid $\left(\mathrm{CH}_{3} \mathrm{CHClCO}_{2} \mathrm{H}\right)$, acetic acid, 2-propanol, sodium hydroxide, sodium hydrogen carbonate, and

\footnotetext{
$\overline{{ }^{4} \text { Introduction }}$ of charges into fibrous systems through physisorption has been shown to be less effective than chemical grafting (Naderi et al. 2016b).
}

methanol were purchased from Sigma Aldrich (Sweden).

Poly-dimethyldiallylammonium chloride (DMDAAC, trade name: Alcofix 109) with a charge density of $6.2 \mathrm{meq} / \mathrm{g}$ was purchased from Allied Colloids Ltd (UK). Before use, the polymer was ultrafiltrated with a Millipore Labscale TFF ultrafiltration unit using a Pellicon XL membrane (cut-off $5 \times 10^{5}$ ) to remove low molecular weight material. Size exclusion chromatography (SEC) was used to estimate the molecular mass of the polymers. The weight averaged molecular weight and polydispersity index of DMDAAC were $550 \mathrm{~kg} / \mathrm{mol}$ and 3.6 , respectively. Polyethylenimine (PEI) with a molecular weight of about $60 \mathrm{~kg} / \mathrm{mol}$ (supplied by Arcos organics, US) was used as received.

Green muscovite mica was obtained from AXIM Enterprises (New York, US). Deionized water was used through-out this study.

\section{Alkoxylation of pulp}

Alkoxylation of pulp was conducted by two different reagents, monochloroacetic acid (MCA) and 2-chloropropionic acid (CPA). The general description of the alkoxylation process is as follows.

The never-dried fibers were dispersed in water at 10,000 revolutions using an ordinary laboratory blender. The fibers were then solvent-exchanged to ethanol by washing the fibers in $1 \mathrm{~L}$ of ethanol four times with a filtering step in between.

The fibers were then impregnated for $30 \mathrm{~min}$ with a solution of the reagent dispersed in isopropanol. Subsequently, the fibers were added in portions to a solution of $\mathrm{NaOH}$ dissolved in methanol and isopropanol, that had been heated just below its boiling temperature in a reaction vessel fitted with a condenser. The alkoxylation reaction was allowed to continue for 1 or $3 \mathrm{~h}$, depending on the reagent (Table 1).

Following the completion of the reaction, the fibers were filtered and washed in three steps. First, the fibers were washed with deionized water. Thereafter, the fibers were washed with acetic acid $(0.1 \mathrm{M})$ and finally with water. The fibers were then impregnated with $\mathrm{NaHCO}_{3}$ solution $(4 \% \mathrm{w} / \mathrm{w})$ for $60 \mathrm{~min}$ in order to convert the carboxyl groups to their sodium form. Then, the fibers were washed with water and drained on a Büchner funnel. 
Table 1 The reaction compositions for the different alkoxylation reactions of this study

\begin{tabular}{|c|c|c|}
\hline & Pulp $_{\text {MCA }}$ & Pulp \\
\hline Pulp (g) & 30 & 30 \\
\hline $\operatorname{MCA}(\mathrm{g})$ & 2.9 & \\
\hline CPA (g) & & 27.3 \\
\hline $\mathrm{NaOH}(\mathrm{g})$ & 4.4 & 14.1 \\
\hline 2-propanol (g) & 535 & 501 \\
\hline Ethanol (g) & 120 & 120 \\
\hline Methanol (g) & 108 & 108 \\
\hline Heating time (h) & 1 & 3 \\
\hline Reaction yield $(\%)^{\mathrm{a}}$ & 67 & 8 \\
\hline Charge $_{\text {Cond. }}(\mu \mathrm{eq} / \mathrm{g})$ & $646 \pm 10$ & $679 \pm 10$ \\
\hline Charge $_{\mathrm{Tit}} /$ Charge $_{\text {Cond. }}$ & $0.06 \pm 0.01$ & $0.09 \pm 0.02$ \\
\hline $\mathrm{WRV}_{\text {N.d. }}(\mathrm{g} / \mathrm{g})$ & $2.24 \pm 0.04$ & $3.14 \pm 0$ \\
\hline $\mathrm{WRV}_{\text {Redisp. }}(\mathrm{g} / \mathrm{g})$ & $1.51 \pm 0$ & $2.70 \pm 0.08$ \\
\hline
\end{tabular}

"MCA" denotes monochloroacetic acid, and "CPA" 2-chloropropionic acid. The table also contains the bulk and surface charge densities of the systems, which have been obtained by conductometric (Cond.) measurement and polyelectrolyte titration (Tit.). Water retention values (WRV) were measured on never-dried (N.d.) and redispersed (Redisp.) alkoxylated pulps

a Reaction yield is defined as the molar ratio between the conductometrically determined charge density, and the molar ratio of the amount of reagent and glucopyranose units

The total charge of fibres, in sodium counter-ion form, was determined by means of conductometric titration based on a method developed by Katz et al. (1984).

\section{Production of NFC systems}

The alkoxylated pulps were dispersed in water [to a consistency of about $2 \%(\mathrm{w} / \mathrm{w})$ ] by a propeller mixer for $1 \mathrm{~h}$. The suspensions were thereafter homogenized (Microfluidizer M-110EH, Microfluidics Corp., USA) by passing the suspensions one time at a pressure of 1700 bar through two Z-shaped chambers with diameters of 200 and $100 \mu \mathrm{m}$, respectively. The products were thereafter kept refrigerated $\left(\right.$ at $5{ }^{\circ} \mathrm{C}$ ) until used.

Surface charge density of fibres obtained by polyelectrolyte titration

The surface charges of the alkoxylated fibres (in their sodium counter-ion form) was determined by using the polyelectrolyte titration method of Horvath et al. (2006); DMDAAC was used in the investigations.

Protocol for drying of NFC and its subsequent redispersion

Nanofibrillated cellulose suspensions (2\% (w/w), $300 \mathrm{~g}$ ) were poured into 2-litre petri dishes, and were dried in an oven at $105{ }^{\circ} \mathrm{C}(18 \mathrm{~h})$. Subsequently, the dried materials were torn into smaller pieces and were equilibrated overnight in deionized water, at a total dry content of $2 \%(\mathrm{w} / \mathrm{w})$. The suspensions were thereafter mixed with a propeller mixer (at $2000 \mathrm{rpm}$ for $2 \mathrm{~min}$ ), and then homogenized (at 20,000 rpm for $30 \mathrm{~s}$ ) using a rotor-stator homogenizer (Kinematica polytron homogenizer PT-3100D, Switzerland).

Water retention value (WRV)

The SCAN-C 62:00 standard was employed for measuring the WRV of the different never-dried pulps, in their sodium counter-ion form.

In part of the investigations, WRV studies were also conducted on oven-dried pulps $\left(105^{\circ} \mathrm{C} / 18 \mathrm{~h}\right)$. In this case, the measurements were preceded by dispersing the pulps in water at 30,000 revolutions using an ordinary laboratory blender.

Preparation of NFC-films

Samples with dry contents of about $0.1 \%(\mathrm{w} / \mathrm{w})$ were prepared by blending appropriate amounts of the concentrated materials with water, and stirring with a magnetic stirrer, for $18 \mathrm{~h}$ at $750 \mathrm{rpm}$. The obtained suspensions were thereafter degassed for $1 \mathrm{~h}$. Films were prepared first through vacuum filtration of the suspension using $0.65 \mu \mathrm{m}$ DVPP filters (supplied by Millipore), and thereafter drying, in constrained form, in an oven for $7 \mathrm{~h}$ at $50{ }^{\circ} \mathrm{C}$.

Tensile strength measurements of films based on NFC

An MTS tensile strength machine with a Teststar IIS controller (MTS, USA) was used in the investigations. The samples were kept at $50 \% \mathrm{RH} / 23{ }^{\circ} \mathrm{C}$, for at least 3 days, before conducting the measurements. The samples were weighted after strips were cut out. The length and width of the strips were 45 and $6 \mathrm{~mm}$, 
respectively; the distance between the grips holding the strips was $30 \mathrm{~mm}$. The strips were then mounted into a tensile strength machine and the mechanical properties were measured at a speed of $100 \% / \mathrm{min}$.

\section{Oxygen permeability measurements}

The oxygen transmission rate (OTR) was monitored with a Mocon Ox-Tran model 2/20 MH System equipped with a coulometric oxygen sensor (Mocon, Minneapolis, USA). The NFC films were mounted in an isolated diffusion cell, where one side of the films is exposed to oxygen (99.95\%) at atmospheric pressure. The oxygen, which permeates through the sample, is transported to a coulometric sensor, where the amount of oxygen is measured. The OTR-value was normalized with respect to the average thickness of the films (measured by scanning electron microscopy) to yield an oxygen permeability (OP) value. The measurements were conducted at $23{ }^{\circ} \mathrm{C}$ and $50 \% \mathrm{RH}$, and at $23{ }^{\circ} \mathrm{C}$ and $80 \% \mathrm{RH}$, respectively.

\section{Rheological studies}

The rheological studies were conducted on samples that after their manufacturing had been stored in a fridge $\left(5^{\circ} \mathrm{C}\right)$ for at least 3 days, and then equilibrated overnight at room temperature.

The investigations were performed using a Kinexus stress controlled rotational rheometer (Malvern Instruments, UK) together with the software rSpace (Malvern Instruments, UK). A standard (ISO 3219/DIN 53019) metal concentric cylinder (bob and cup) geometry with serrated surfaces was used (to minimize slippage effects (Buscall 2010)) in the studies. The height and distance between the serrations were 300 and $1000 \mu \mathrm{m}$, respectively. The diameter and length of the bob were 25 and $37.5 \mathrm{~mm}$, respectively; the diameter and wall height of the cup were 27.5 and $62.5 \mathrm{~mm}$, respectively. A working gap of $9.15 \mathrm{~mm}$ was employed in the measurements. The set experimental temperature was $25^{\circ} \mathrm{C}$; the samples were covered with a protective hood during the measurements.

The NFC samples were sheared at $100 \mathrm{~s}^{-1}$ for $1 \mathrm{~min}$ in the measuring chamber, as a mean to even out the heterogeneities, and then were left to equilibrate for $2 \mathrm{~min}$ before conducting the measurements. Integration time per measuring point was set to $30 \mathrm{~s}$.
Determination of the apparent efficiency of the fibrillation process

Nanofibrillated cellulose samples with a consistency of about $0.02 \%(\mathrm{w} / \mathrm{w})$ were prepared by first blending the concentrated NFC systems with water (using a magnetic stirrer for about $18 \mathrm{~h}$ at $750 \mathrm{rpm}$ ). The diluted systems were thereafter centrifuged at $1000 \mathrm{~g}$ for $15 \mathrm{~min}$, to remove the larger constituents (e.g. residual fibre-fragments). It is noted that in an earlier contribution (Naderi et al. 2014) the apparent network forming concentration of a carboxymethylated NFC was $0.04-0.07 \%(\mathrm{w} / \mathrm{w})$, below which the larger fibre wall fragments were more easily removed by centrifugation.

The concentrations of the suspensions obtained before $\left(c_{b c}\right)$ and after $\left(c_{a c}\right)$ centrifugation were used to estimate the fraction of nano-sized cellulosic materials $\left[\mathrm{c}_{\mathrm{NS}} \%(\mathrm{w} / \mathrm{w})\right]$ as:

$\mathrm{c}_{\mathrm{NS}} \%(\mathrm{w} / \mathrm{w})=\frac{\mathrm{c}_{\mathrm{ac}}}{\mathrm{c}_{\mathrm{bc}}} \times 100$

In Eq. (1) $c_{N S}$ denotes the fraction of entities which have the ability to resist phase separation during centrifugation. This method of analysis assumes that the magnitude of $c_{N S}$ increases with the increasing efficiency of the fibrillation process.

Atomic force microscopy studies

AFM tapping mode imaging was performed with a MultiMode 8 (Bruker, Santa Barbara, CA) in air using soft tapping cantilever with typical values for resonance frequency of $150 \mathrm{kHz}$, spring constant $4.5 \mathrm{~N} / \mathrm{m}$ and tip radius $8 \mathrm{~nm}$ (Tap150, Bruker, Camarillo, CA).

Samples for imaging studies were prepared by using freshly cleaved muscovite mica immersed for $1 \mathrm{~s}$ in PEI solution $\left(0.01 \mathrm{~g} / \mathrm{dm}^{3}\right)$, followed by rinsing with an excess of water, and immersion for $10 \mathrm{~s}$ in NFC suspensions. The surfaces were thereafter rinsed with an excess of water and then gently dried in a flow of filtered nitrogen gas, before the start of the measurements.

Nuclear magnetic resonance (NMR) studies

All samples had a water content of at least $40 \%$ and were packed uniformly in a zirconium oxide rotor. The CP/MAS 13C-NMR spectra were recorded in a Bruker 
Avance III AQS $400 \mathrm{SB}$ instrument operating at $9.4 \mathrm{~T}$. All measurements were carried out at $297( \pm 1) \mathrm{K}$ with a magic angle spinning (MAS) rate of $10 \mathrm{kHz}$. A 4-mm double air-bearing probe was used. Data acquisition was performed using a cross-polarization (CP) pulse sequence, i.e., a 2.95 microseconds proton 90 degree pulse and an 800 microseconds ramped (100-50\%) falling contact pulse, with a $2.5 \mathrm{~s}$ delay between repetitions. A SPINAL64 pulse sequence was used for ${ }^{1} \mathrm{H}$ decoupling. The Hartmann-Hahn matching procedure was based on glycine. The chemical shift scale was calibrated to the TMS-scale (tetramethylsilane, $\left.\left(\mathrm{CH}_{3}\right)_{4} \mathrm{Si}\right)$ by assigning the data point of maximum intensity in the alpha-glycine carbonyl signal to a chemical shift value of 176.03 ppm. 4096 or 32,768 transients were recorded on each sample depending on solids content, leading to acquisition times between 3 and $24 \mathrm{~h}$.

Solid state cross-polarization magic angle spinning carbon-13 nuclear magnetic resonance (CP/MAS ${ }^{13} \mathrm{C}$ NMR) spectra were recorded on NFC samples, and the (dissolving) pulp, for comparison purposes.

The solids contents of NFC samples were increased by first lowering the $\mathrm{pH}$ (by $\mathrm{HCl}$ ) to $2-3$, which induced precipitation. The precipitates were thereafter immersed in deionized water and centrifuged $\left(2 \times 5000 \mathrm{~g}, 1 \mathrm{~h}, 25^{\circ} \mathrm{C}\right)$. Finally, the sediments were dewatered on a glass filter, and packed into an NMR rotor.

\section{Results}

In Table 1, the optimized compositions of the different alkoxylation recipes of this report are summarized. In what follows, the alkoxylated pulps will be denoted as $\mathrm{Pulp}_{\alpha}$, where $\alpha$ refers to the type of reagent (MCA or CPA). In the similar manner, NFCs based on the pulps will be referred to by $\mathrm{NFC}_{\alpha}$.

Table 1 reveals that the efficiency of the alkoxylation process is significantly lower when CPA is used; as the amounts of employed reagent and $\mathrm{NaOH}$ are larger, and the reaction time is significantly longer than in the corresponding MCA-based process. The lower reactivity of CPA may be explained by steric factors, due to the larger size of CPA and possibly also by an inherent lower reactivity at the conditions used. In this context, it is noted (but not shown) that longer homologs of the chloro-substituted aliphatic acids display very little reactivity ${ }^{5}$ at similar reaction conditions.

Table 1 further reveals that the $\mathrm{WRV}_{\text {N.d. }}$ of neverdried Pulp $\mathrm{CPA}_{\mathrm{C}}$ is significantly higher than that of Pulp MCA. $_{\text {. }}$

In Fig. 1, the morphologies of never-dried $\mathrm{NFC}_{\mathrm{MCA}}$ and $\mathrm{NFC}_{\mathrm{CPA}}$ measured by $\mathrm{AFM}$-imaging are presented. It was found that the lengths (L) and widths (w) of the nanofibrillated systems were about the same $(\mathrm{L} \approx 1 \mu \mathrm{m}, \mathrm{w} \approx 5 \mathrm{~nm}$ ); however, as will be shown, the overall properties of the NFCs are generally very different. An NFC system's properties are defined by all the system constituents (nanofibrils, fibre fragments, etc.) and their relative proportions. It is emphasized that the AFM-imaging studies (as performed in this study) focus on the smallest particles that remain in the supernatant during the centrifugation employed as part of the sample preparation.

In Table 2, the apparent shear viscosities (measured at $\dot{\gamma}=1 \mathrm{~s}^{-1}$ ) of the NFCs based on the alkoxylated pulps of Table 1 are presented. As it can be seen, the viscosity of $\mathrm{NFC}_{\mathrm{CPA}}$ is about $30 \%$ higher than the viscosity of $\mathrm{NFC}_{\mathrm{MCA}}$.

In the same table, the apparent fraction of nanosized materials $\left(\mathrm{c}_{\mathrm{NS}}\right)$ and tensile strength index (TSI) values of the different NFCs, in never-dried state, are also shown. The $\mathrm{c}_{\mathrm{NS}}$ of $\mathrm{NFC}_{\mathrm{CPA}}$ is about $30 \%$ higher than for $\mathrm{NFC}_{\mathrm{MCA}}$; hence, the trend in the $\mathrm{c}_{\mathrm{NS}}$-values is similar to the observed trend in the viscosities of the nanofibrillated systems. However, the difference in the magnitude of TSI of the different NFCs is insignificant. Similar observations have been made earlier (Naderi et al. 2015a, 2016a). The observations can be rationalized by the high sensitivity of rheological properties towards the degree of fibrillation, and the insensitivity of TSI towards the degree of fibrillation above a critical value. The critical degree of fibrillation of a alkoxylated NFC similar to the $\mathrm{NFC}_{\mathrm{MCA}}$ of this report has been shown to be in the order of 30-40\% (Naderi et al. 2015a).

In Table 3, the oxygen permeability (OP) values for films produced from never-dried NFCs are summarized. The $\mathrm{NFC}_{\mathrm{CPA}}$ displays significantly higher OPvalues compared to $\mathrm{NFC}_{\mathrm{MCA}}$. The trends in the OPvalues can be rationalized by considering the relative magnitudes of the WRVs of the never-dried and

5 Also, the linear 3-chloropropionic acid displays very low reactivity. 
Fig. 1 AFM-imaging of centrifuged-fractionated never-dried $\mathrm{NFC}_{\mathrm{MCA}}$ (a) and never-dried $\mathrm{NFC}_{\mathrm{CPA}}$ (b). The scan size of the images is $5 \times 5 \mu \mathrm{m}$
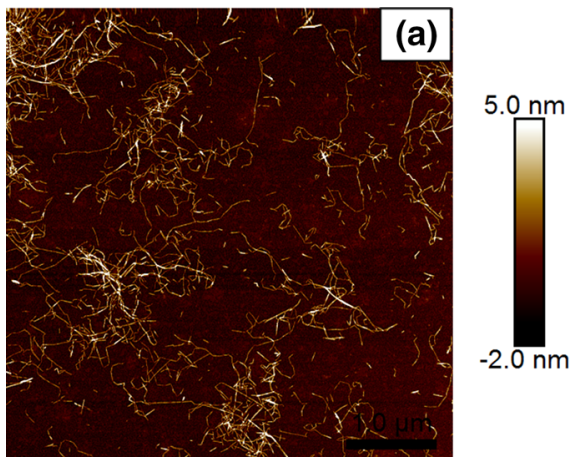

(b)

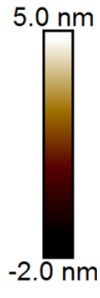

Table 2 The shear viscosity (measured at $\dot{\gamma}=1 \mathrm{~s}^{-1}$ ), tensile strength index (TSI) and fraction of nano-sized materials $\left(\mathrm{c}_{\mathrm{NS}}\right)$ of never-dried (N.d.) and redispersed (Redisp.) NFCs

\begin{tabular}{|c|c|c|c|c|c|c|}
\hline & Viscosity $_{\text {N.d. }}($ Pa.s $)$ & $\frac{\text { Viscosity }_{\text {Redisp. }}}{\text { Viscosity }_{N . d .}}$ & $\mathrm{TSI}_{\mathrm{N} . \mathrm{d} .}(\mathrm{kNm} / \mathrm{kg})$ & $\frac{T S I_{\text {Redisp. }}}{T S I_{N . d .}}$ & $\mathrm{c}_{\text {NS-N.d. }}(\%)$ & $\frac{c_{N S-R e d i s p}}{c_{N S-N . d .}}$ \\
\hline $\mathrm{NFC}_{\mathrm{MCA}}$ & $87 \pm 4$ & $0.1 \pm 0.02$ & $166 \pm 16$ & $0.77 \pm 0.1$ & $31 \pm 1$ & $0.1 \pm 0.07$ \\
\hline $\mathrm{NFC}_{\mathrm{CPA}}$ & $116 \pm 6$ & $0.9 \pm 0.02$ & $170 \pm 9$ & $0.90 \pm 0.08$ & $41 \pm 1$ & $0.72 \pm 0.06$ \\
\hline
\end{tabular}

redispersed alkoxylated pulps, which suggest that the systems with higher WRVs swell more in humid conditions $(50 \% / 80 \% \mathrm{Rh})$ compared to systems that have lower WRVs. This reasoning finds support in a recent report (Naderi et al. 2016b) wherein it was shown that a low-charged enzymatically pre-treated $\mathrm{NFC}(\approx 30 \mu \mathrm{eq} / \mathrm{g})$ had about an order of magnitude lower OP-value compared to a MCA-alkoxylated NFC $(\approx 600 \mu \mathrm{eq} / \mathrm{g})$, similar to the findings of this report.

The appearances of the dried NFCs after $24 \mathrm{~h}$ of soaking in water are displayed in Fig. 2a, b. As it can be seen, the dried $\mathrm{NFC}_{\mathrm{MCA}}$ displays little swelling in water, but the dried $\mathrm{NFC}_{\mathrm{CPA}}$ shows a significant swelling. Noticeable swelling of $\mathrm{NFC}_{\mathrm{CPA}}$ occurred within minutes, after immersion in water.

Figure $2 \mathrm{c}$ displays a representative picture of the appearance of the $\mathrm{NFC}_{\mathrm{CPA}}$ after the propeller-mixing step in the redispersion process. As it can be seen, a highly viscous suspension is obtained with low amount of shearing; this observation was, however, not made with $\mathrm{NFC}_{\mathrm{MCA}}$. Of interest to these observations are the WRVs of the dried and redispersed $\left(\mathrm{WRV}_{\text {Redisp. }}\right)$ alkoxylated pulps that have been displayed in Table 1. As it can be seen, the $\mathrm{WRV}_{\text {Redisp. }}$ of Pulp $_{\mathrm{CPA}}$ is significantly higher than the equivalent for

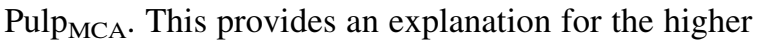
swelling- and redispersion ability of $\mathrm{NFC}_{\mathrm{CPA}}$ than $\mathrm{NFC}_{\mathrm{MCA}}$.
Figure $2 \mathrm{~d}$ displays a highly diluted $\mathrm{NFC}_{\mathrm{CPA}}$, after the homogenisation step in the redispersion process. The presence of distinguishable particles indicates an incomplete redispersion. An efficient redispersion is dependent on an efficient flow of the dispersion through the homogenizer. During the homogenization of $\mathrm{NFC}_{\mathrm{CPA}}$ the flow efficiency was observed to diminish more rapidly than for $\mathrm{NFC}_{\mathrm{MCA}}$, as the viscosity of the former evolved more rapidly and to a higher value (see Table 2). Nevertheless, as it can be seen in Tables 2 and 3 the redispersed $\mathrm{NFC}_{\mathrm{CPA}}$ regains most of the never-dried properties-which is not observed for the redispersed $\mathrm{NFC}_{\mathrm{MCA}}$.

It has been proposed (Naderi et al. 2016a) that more spatially homogeneous distribution of the charges throughout the fibre wall (displayed by an increase in the Charge $\mathrm{Tit}_{\mathrm{Tit}} /$ Charge $_{\text {Cond. }}$ ratio) has beneficial effects on the properties of NFCs. The claim was demonstrated by the study of the degree of fibrillation of a MCA-alkoxylated NFC that had been produced by the inclusion of small amounts of water $(2.5-4 \%(w / w))$ in the reaction medium; the impact of the charge distribution on redispersion properties was, however, not studied. Based on the findings, it could be hypothesized that the improved never-dried and redispersed properties of $\mathrm{NFC}_{\mathrm{CPA}}$ might be due to a more homogeneous distribution of the charges throughout the fibre wall. It is recapitulated that a 
Table 3 Oxygen permeability (OP) values of NFC-films based on never-dried (N.d.) and redispersed (Redisp.) NFCs

\begin{tabular}{lllll}
\hline & $\begin{array}{l}\mathrm{OP}\left(23{ }^{\circ} \mathrm{C} / 50 \% \mathrm{RH}\right) \\
\left(\mathrm{cm}^{3} \mu \mathrm{mm}^{-2} \mathrm{~d}^{-1} \mathrm{kPa}^{-1}\right)\end{array}$ & $\begin{array}{l}O P_{\text {Redisp. }} \\
O P_{\text {N.d. }}\end{array}$ & $\begin{array}{l}\mathrm{OP}\left(23{ }^{\circ} \mathrm{C} / 80 \% \mathrm{RH}\right) \\
\left(\mathrm{cm}^{3} \mu \mathrm{mm}^{-2} \mathrm{~d}^{-1} \mathrm{kPa}^{-1}\right)\end{array}$ & $\begin{array}{l}O P_{\text {Redisp. }} \\
O P_{\text {N.d. }}\end{array}$ \\
\hline $\mathrm{NFC}_{\mathrm{MCA}}$ & $0.4 \pm 0.06$ & $1.1 \pm 0.2$ & $9.8 \pm 1.6$ & $1.2 \pm 0.2$ \\
$\mathrm{NFC}_{\mathrm{CPA}}$ & $1.0 \pm 0.2$ & $0.7 \pm 0.2$ & $24.6 \pm 4.0$ & $1.3 \pm 0.3$ \\
\hline
\end{tabular}
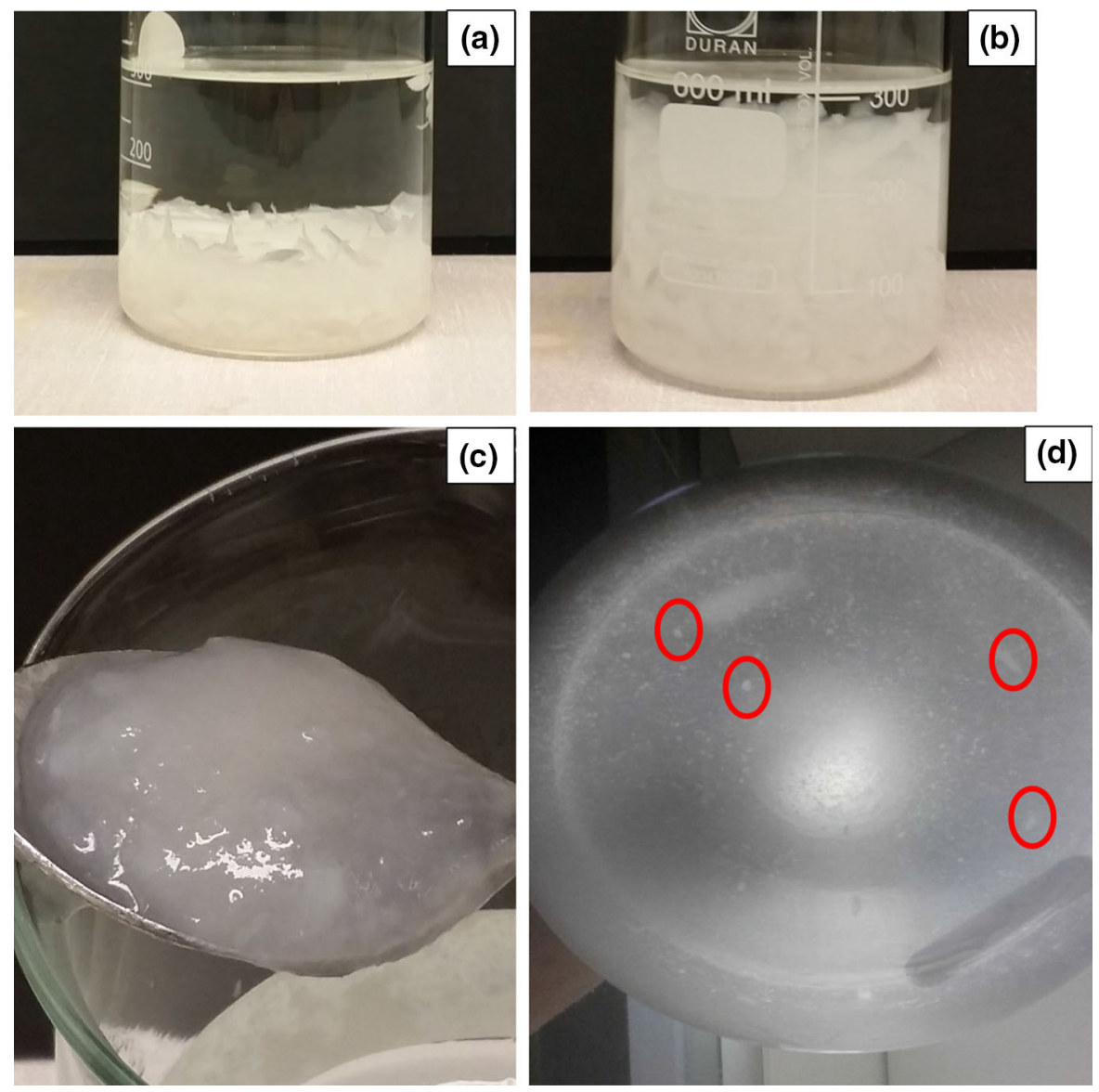

Fig. 2 The states of dried NFCs after immersion in water for $24 \mathrm{~h} ; \mathrm{NFC}_{\mathrm{MCA}}(\mathbf{a})$ and $\mathrm{NFC}_{\mathrm{CPA}}(\mathbf{b})$. c A representative picture of the state of $\mathrm{NFC}_{\mathrm{CPA}}$ after propeller mixing in the redispersion process. $\mathbf{d}$ A representative picture (seen from the bottom of the

significant higher amount of $\mathrm{NaOH}$ ( $>3$ times) is used when CPA is employed instead of MCA (Table 1), which can enhance the swelling of the fibre. However, as it can be seen in Table 1, the Charge ${ }_{\text {Tit. }} /$ Charge $_{\text {Cond. }}$. ratios of Pulp $\mathrm{PCA}_{\mathrm{C}}$ and Pulp $\mathrm{PPA}_{\mathrm{CP}}$ are about the same.

To further investigate the hypothesis that the spatial distribution of charges in the fibre wall could be different between Pulp $\mathrm{pCA}_{\mathrm{MCA}}$ and Pulp $\mathrm{p}_{\mathrm{CPA}}$, due to the different $\mathrm{NaOH}$ charges used, MCA-based pulps were

container) of diluted $(0.02 \%(\mathrm{w} / \mathrm{w})) \mathrm{NFC}_{\mathrm{CPA}}$ after the homogenization-treatment in the redispersion process, showing that the suspension contains undispersed particles. A few of these particles have been highlighted with rings

also prepared via two different routes (Table 4). During the first route MCA-alkoxylation was conducted using the same amount of $\mathrm{NaOH}$ and heating time as for the production of Pulp $\mathrm{PPA}_{\mathrm{CP}}$. Never-dried and redispersed properties of the pulp (denoted by MCAHigh- $\mathrm{NaOH}$ ) and the corresponding NFC have been summarized in Table 5. As it can be seen, the Charge $_{\text {Tit. }}$ /Charge Cond. $_{\text {of Pulp }}$ MCA-High-NaOH is similar to that of Pulp CPA $_{\text {(and Pulp }}$ MCA). However, the WRV 
Table 4 The reaction compositions of different MCA-based alkoxylation reactions

\begin{tabular}{lll}
\hline & MCA-High-NaOH & MCA- $\mathrm{H}_{2} \mathrm{O}$ \\
\hline Pulp (g) & 30 & 30 \\
MCA (g) & 2.9 & 2.7 \\
NaOH (g) & 14 & 4.4 \\
2-propanol (g) & 535 & 518 \\
Ethanol (g) & 120 & 120 \\
Methanol (g) & 108 & 108 \\
Water (g) & & 34 \\
Heating time (h) & 3 & 1 \\
\hline
\end{tabular}

In the composition denoted by MCA-High-NAOH, the amount of $\mathrm{NaOH}$ and the reaction time are the same as in the alkoxylation reaction that was employed for the production of Pulp $_{\text {CPA }}$. MCA- $\mathrm{H}_{2} \mathrm{O}$ denotes a alkoxylation process that is

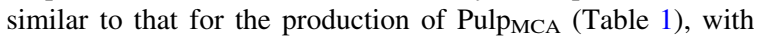
the exception of including $4 \%(\mathrm{w} / \mathrm{w})$ of water in the reaction medium

of Pulp MCA-High-NaOH is significantly lower than

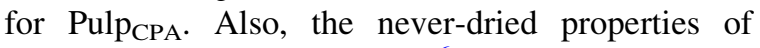
$\mathrm{NFC}_{\text {MCA-High-NaOH }}$ are inferior ${ }^{6}$ to those of $\mathrm{NFC}_{\mathrm{CPA}}$, and evidently (Table 5) $\mathrm{NFC}_{\mathrm{MCA}-\mathrm{High}-\mathrm{NaOH}}$ cannot be redispersed as efficiently ${ }^{7}$ as $\mathrm{NFC}_{\mathrm{CPA}}$.

During the second route, alkoxylation was conducted by the addition of $4 \%(\mathrm{w} / \mathrm{w})$ of water to the reaction medium (Table 4). As it can be seen in Table 5, the magnitude of Charge $\mathrm{Tit}_{\mathrm{Tit}} / \mathrm{Charge}_{\mathrm{Cond}}$. of Pulp ${\mathrm{MCA}-\mathrm{H}_{2} \mathrm{O}}_{\text {is about the same as Pulp }}$ CPA (and Pulp MCA-High-NaOH$)$. The never-dried properties of $\mathrm{NFC}_{\mathrm{MCA}-\mathrm{H}_{2} \mathrm{O}}$ are, however, higher than those of $\mathrm{NFC}_{\mathrm{CPA}}$, but the properties of the redispersed $\mathrm{NFC}_{\mathrm{CPA}}$ are superior to those of $\mathrm{NFC}_{\mathrm{MCA}-\mathrm{H}_{2} \mathrm{O}}$ No apparent swelling of the dried $\mathrm{NFC}_{\mathrm{MCA}-\mathrm{H}_{2} \mathrm{O}}$ was observed, after $24 \mathrm{~h}$ of immersion in water (data not shown).

Hence, the results (the similar Charge Tit. $_{\text {I }}$ Charge $_{\text {Cond. }}$ ratios of the different MCA-based pulps and Pulp $\mathrm{PPA}_{\mathrm{CP}}$, but their dissimilar redispersion properties) indicate that a more homogeneous distribution of the charges in the CPA-based NFC can be ruled out as

\footnotetext{
${ }^{6}$ Limiting viscosity measurements (ISO 5351:2010) on the carboxymethylated pulp revealed a significantly lower viscosity

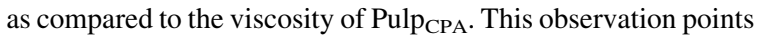
to the significant hydrolysis of the former system, which provides a possible explanation for the observations.

7 It was noted (but not shown) that the visual appearance of the dried $\mathrm{NFC}_{\mathrm{MCA}-\mathrm{High}-\mathrm{NaOH}}$ after soaking in water for $24 \mathrm{~h}$ resembles that of $\mathrm{NFC}_{\mathrm{MCA}}$.
}

Table 5 The never-dried and redispersed properties of pulps and corresponding NFCs based on the alkoxylation processes of Table 4

\begin{tabular}{|c|c|c|}
\hline & MCA-High- $\mathrm{NaOH}$ & $\mathrm{MCA}-\mathrm{H}_{2} \mathrm{O}$ \\
\hline Charge $_{\text {Cond. }}(\mu \mathrm{eq} / \mathrm{g})$ & $630 \pm 30$ & $642 \pm 19$ \\
\hline Charge $_{\text {Tit. }} /$ Charge $_{\text {Cond. }}$ & $0.08 \pm 0.01$ & $0.08 \pm 0.01$ \\
\hline $\mathrm{WRV}_{\text {N.d. }}(\mathrm{g} / \mathrm{g})$ & $2.4 \pm 0.05$ & $9.4^{\mathrm{a}} \pm 0.3$ \\
\hline WRV $_{\text {Redisp. }}(\mathrm{g} / \mathrm{g})$ & $1.9 \pm 0$ & $1.8 \pm 0.03$ \\
\hline Viscosity (Pa.s) & $48 \pm 5$ & $155 \pm 24$ \\
\hline$\frac{\text { Viscosity }_{\text {Redisp. }}}{\text { Viscosity }_{\text {N.d. }}}$ & $0.2 \pm 0.03$ & $0.3 \pm 0.02$ \\
\hline $\mathrm{TSI}_{\text {N.d. }}(\mathrm{kNm} / \mathrm{kg})$ & $137 \pm 6$ & $169 \pm 9$ \\
\hline$\frac{T S I_{\text {Redisp. }}}{T S I_{N . d .}}$ & $0.82 \pm 0.06$ & $0.67 \pm 0.06$ \\
\hline $\mathrm{C}_{\mathrm{NS}}(\%)$ & $28 \pm 3$ & $47 \pm 5$ \\
\hline$\frac{c_{N S-R e d i s p}}{c_{N S-N . d .}}$ & $0.35 \pm 0.06$ & $0.38 \pm 0.08$ \\
\hline
\end{tabular}

${ }^{a}$ The extremely high value is due to the clogging of the wirecloth that is used in WRV studies (Naderi et al. 2016a). The viscosity was measured at $\dot{\gamma}=1 \mathrm{~s}^{-1}$

a major contribution to the superior redispersion properties of the system, as compared to when MCA is used.

The spectra in Fig. 3 show an impact on the cellulose supra-molecular structure as a result of chemical modification with MCA and CPA. In order to interpret the observable spectral changes the $\mathrm{C} 4$ spectra region (80-92 ppm) was studied. Figure 4 displays the $\mathrm{C} 4$ spectral region of samples $\mathrm{NFC}_{\mathrm{MCA}}$ $\mathrm{NFC}_{\mathrm{MCA}-\mathrm{H}_{2} \mathrm{O}}$ and $\mathrm{NFC}_{\mathrm{CPA}}$ superimposed. The interpretation of the $\mathrm{C} 4$ spectral region consists of two main regions; signals from $\mathrm{C} 4$ atoms in polymers located in the highly order (crystalline) fibril interior (86-92 ppm), and signals from $\mathrm{C} 4$ atom in polymers constituting the fibril surfaces (80-86 ppm). In highly ordered samples, it is possible to distinguish between different kinds of fibril surface, those interacting with neighboring fibrils ("inaccessible surfaces") and those interacting with interstitial water ("accessible surface"; Larsson et al. 1997; Wickholm et al. 1998). However, the deterioration of spectral detail accompanied by the MCA and CPA modifications makes a detailed analysis of the surface signals difficult.

An alternate and simpler analysis was performed by arbitrarily scaling superimposed spectra in such a fashion that the maximum signal height in the crystalline $\mathrm{C} 4$ region (86-92 ppm) became the same (Fig. 4). The rationale for this was that the fibril surfaces, which are the primary target for the chemical 


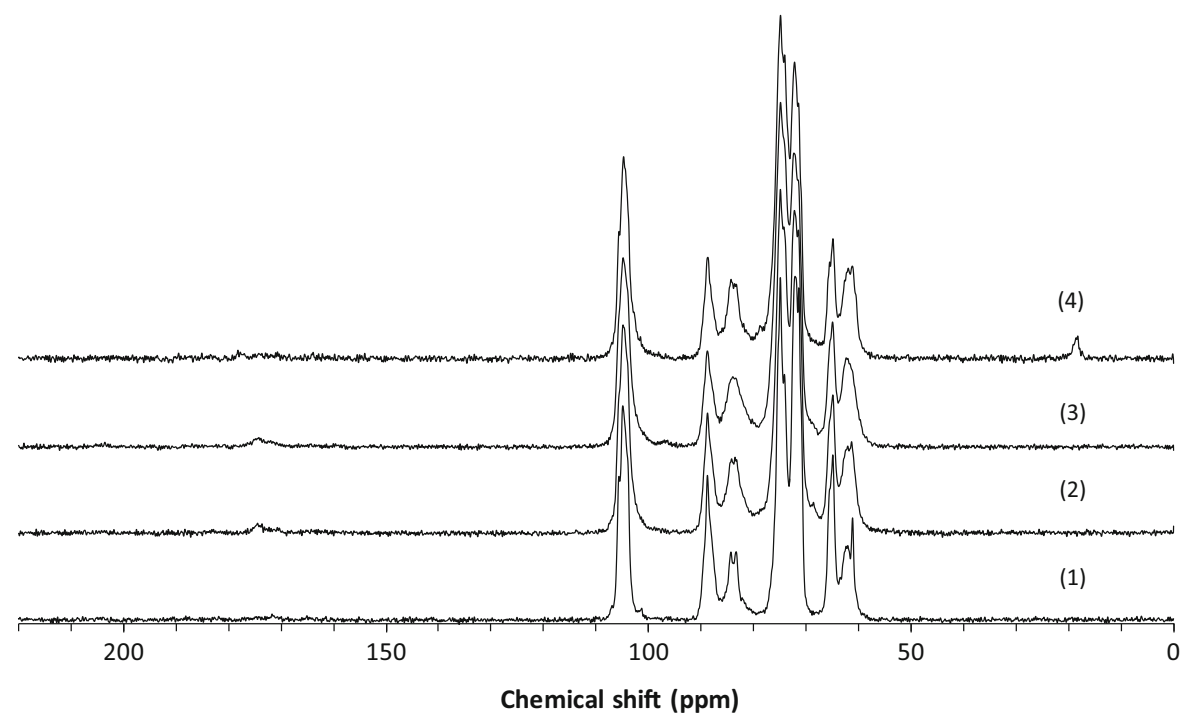

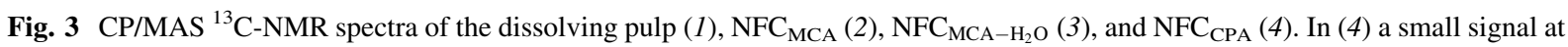
$18 \mathrm{ppm}$ is visible, which originates from the methyl group of the CPA moiety

Fig. 4 The $\mathrm{C} 4$ region of spectra recorded on samples $\mathrm{NFC}_{\mathrm{MCA}}($ black),

$\mathrm{NFC}_{\mathrm{MCA}-\mathrm{H}_{2} \mathrm{O}}$ (blue) and $\mathrm{NFC}_{\mathrm{CPA}}$ (red) superimposed. The spectra were arbitrarily scaled to make the maximum signal height in the $\mathrm{C} 4$ crystalline region (86-92 ppm) similar

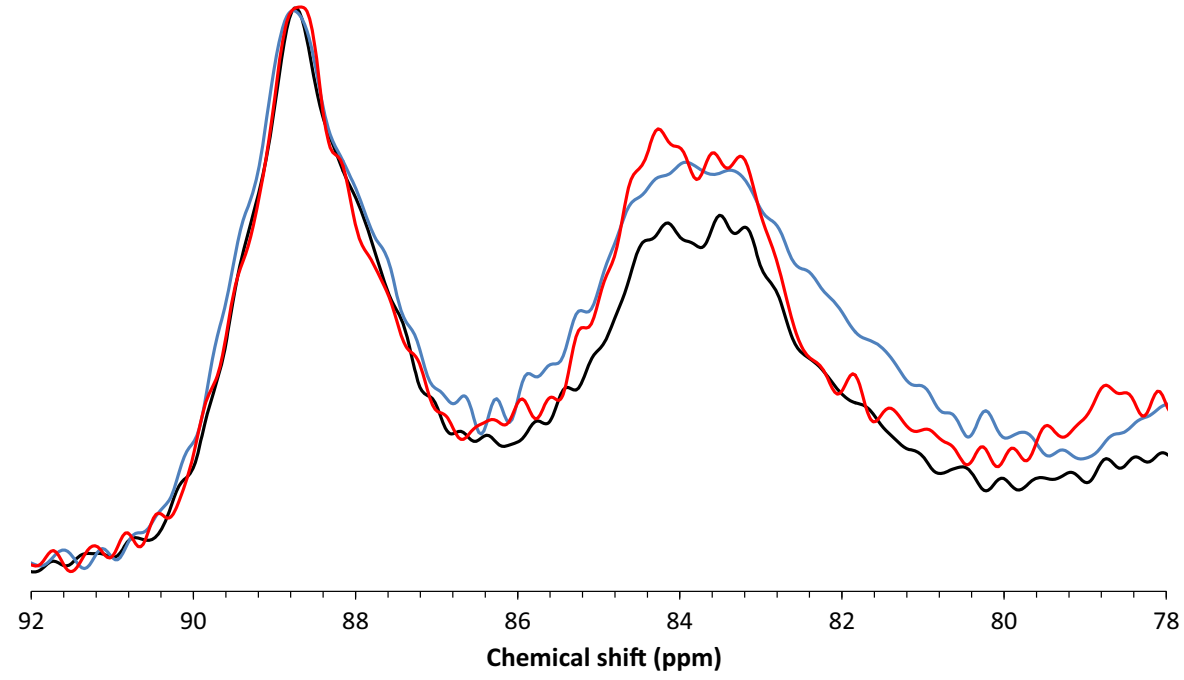

modifications, are believed to be more affected by chemical modification with MCA and CPA than the fibril interior. The scaling of spectra makes it possible to qualitatively assess the relative amount of accessible fibril surfaces. Figure 4 shows that after scaling, a reasonable agreement in integrated signal area was also obtained in the crystalline spectral region. Significant differences in the relative amounts of accessible fibril surface areas were observed among $\mathrm{NFC}_{\mathrm{MCA}}$ and $\mathrm{NFC}_{\mathrm{CPA}}$. The results further indicate that $\mathrm{NFC}_{\mathrm{CPA}}$ and $\mathrm{NFC}_{\mathrm{MCA}-\mathrm{H}_{2} \mathrm{O}}$ exposed more accessible fibril surfaces than $\mathrm{NFC}_{\mathrm{MCA}}$. This was interpreted as being consistent with an increased degree of fibrillation of $\mathrm{NFC}_{\mathrm{CPA}}$ and $\mathrm{NFC}_{\mathrm{MCA}-\mathrm{H}_{2} \mathrm{O}}$; which is supported by the higher viscosity and the $\mathrm{c}_{\mathrm{NS}^{-}}$ value of system (Table 2). In addition, $\mathrm{NFC}_{\mathrm{MCA}-\mathrm{H}_{2} \mathrm{O}}$ also showed a significant signal broadening at about $82 \mathrm{ppm}$. This was interpreted as being the result of an increased variability in the state of order of the

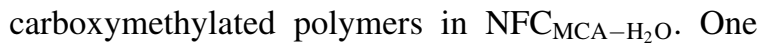
possible cause for such a signal broadening can be a larger degree of heterogeneity in the degree of substitutions (D.S.) of the systems. However, the spectra did not allow for any distinction between types 
of heterogeneity. Whether it is a heterogeneity in D.S. for individual fibrils (patches of low and high charge), or differences in D.S. between individual fibrils with a low total charge mixed with fibrils of a high total charge could not be inferred from spectra. However, the difficulty encountered when determining WRV for $\mathrm{NFC}_{\mathrm{MCA}-\mathrm{H}_{2} \mathrm{O}}$ (Table 5) suggests that more fibrils were liberated from $\mathrm{NFC}_{\mathrm{MCA}-\mathrm{H}_{2} \mathrm{O}}$ (causing the clogging) possibly indicating that the D.S. heterogeneity was mainly due to a variability in total charge between individual fibrils.

It serves to mention that the technique used to record CP/MAS ${ }^{13} \mathrm{C}-\mathrm{NMR}$ spectra is designed to be sensitive to signals originating from rigid structures, and may discriminate signals origination from parts of a sample with high molecular mobility. Spectra recorded on isolated cellulose I has been shown to be quantitative at long enough contact times (Wickholm et al. 1998), but in the case of highly charged $\beta$ $(1,4)$-D-glucan polymers, e.g. highly charged fibrils where surface polymers approach their solubility limit, there is a risk of signal discrimination. Hence, it is possible that at high charge densities CP/MAS ${ }^{13} \mathrm{C}$-NMR spectroscopy fails to detect representative signal intensities for all carbon-13 atoms present in the sample. CP/MAS ${ }^{13} \mathrm{C}$-NMR studies performed on TEMPO-oxidized pulps have indicated that such signal discrimination occurs, but that the effect is small effect even at charge densities up to $1280 \mathrm{ueq} / \mathrm{g}$ (Sjöstedt et al. 2015).

It is well-known that introduction of alien entities viz. charged groups into cellulosic materials reduces the cohesive forces among the nanofibrils, which assists the delamination process in the NFC production. It is also known that introduction of charged groups reduces the hornification effect (Jayme 1944), an effect which diminishes among others the water retention ability of the fibers and the tensile strength properties of corresponding sheets. The investigations revealed that the WRV-value of the CPA-treated pulp is reduced to a lesser extent than the MCA-treated pulp (Table 1) upon drying, and that drying has a lesser impact on the properties $\mathrm{NFC}_{\mathrm{CPA}}$ compared to $\mathrm{NFC}_{\mathrm{MCA}}$. Based on these observations (and discarding dissimilar charge distributions between the different systems as a major contribution), it might be hypothesized that increasing the size of the charged groups leads to an amplified steric interaction, which in turn reduces the cohesive forces among the nanofibrils, in never-dried state, and extent of hornification upon drying.

\section{Conclusions}

The impact of the size of the charged group on the properties of alkoxylated NFCs was investigated. It was found that the employment of the larger 2-chloropropionic acid (CPA) reagent leads to improved properties, e.g. higher fraction of nano-sized materials $\left(\mathrm{c}_{\mathrm{NS}}\right)$ and easier redispersion after drying, as compared to when monochloroacetic acid (MCA) is employed.

Nanofibrillated cellulose products with high degree of fibrillation and the ability to regain most of their original properties are attractive for several industrial applications. This report proposes a route for achieving these properties, based on the hypothesis that the hornification of the nanofibrils can be reduced by increasing the size of the charged groups.

Acknowledgments Gunborg Glad-Nordmark, Åsa Engström and Lars-Åke Hammar are thanked for their competent supporting work. BillerudKorsnäs, Borregaard, Fibria, Hansol Paper, Holmen, ITC, Mercer, Stora Enso, Södra and Research Institutes of Sweden AB are acknowledged for their financial support.

Open Access This article is distributed under the terms of the Creative Commons Attribution 4.0 International License (http:// creativecommons.org/licenses/by/4.0/), which permits unrestricted use, distribution, and reproduction in any medium, provided you give appropriate credit to the original author(s) and the source, provide a link to the Creative Commons license, and indicate if changes were made.

\section{References}

Buscall R (2010) Letter to the editor: wall slip in dispersion rheometry. J Rheol 54(6):1177-1183. doi:10.1122/1. 3495981

Dufresne A (2013) Nanocellulose: a new ageless bionanomaterial. Mater Today 16(6):220-227. doi:10.1016/j.mattod. 2013.06.004

Eyholzer C, Bordeanu N, Lopez-Suevos F, Rentsch D, Zimmermann T, Oksman K (2010) Preparation and characterization of water-redispersible nanofibrillated cellulose in powder form. Cellulose 17:19-30

Håkansson KM, Fall AB, Lundell F, Yu S, Krywka C, Roth SV, Santoro G, Kvick M, Prahl Wittberg L, Wågberg L, Söderberg LD (2014) Hydrodynamic alignment and 
assembly of nanofibrils resulting in strong cellulose filaments. Nat Commun 5:4018. doi:10.1038/ncomms5018

Horvath AE, Lindström T, Laine J (2006) On the indirect polyelectrolyte titration of cellulosic fibers. Conditions for charge stoichiometry and comparison with ESCA. Langmuir 22(2):824-830. doi:10.1021/la052217i

Isogai A, Saito T, Fukuzumi H (2011) TEMPO-oxidized cellulose nanofibers. Nanoscale 3(1):71-85. doi:10.1039/ c0nr00583e

Jayme G (1944) Mikro-Quellungsmessungen an Zellstoffen. Wochenbl Papierfabr 6:187-194

Katz S, Beatson RP, Scallan AM (1984) The determination of strong and weak acidic groups in sulfite pulps. Sven Papperstidn 87:R48-R53

Larsson PT, Wickholm K, Iversen T (1997) A CP/MAS ${ }^{13} \mathrm{C}$ NMR investigation of molecular ordering in celluloses. Carbohyd Res 302(1):19-25. doi:10.1016/S0008-6215 (97)00130-4

Lindström T, Aulin C, Naderi A, Ankerfors M (2014) Microfibrillated cellulose. Encyclopedia of polymer science and technology, pp 1-34

Naderi A, Lindström T, Pettersson T (2014) The state of carboxymethylated nanofibrils after homogenization-aided dilution from concentrated suspensions: a rheological perspective. Cellulose 21(4):2357-2368. doi:10.1007/ s10570-014-0329-9

Naderi A, Lindström T, Sundström J (2015a) Repeated homogenization, a route for decreasing the energy consumption in the manufacturing process of carboxymethylated nanofibrillated cellulose? Cellulose 22(2):11471157. doi:10.1007/s10570-015-0576-4

Naderi A, Lindström T, Sundström J, Flodberg G (2015b) Can redispersible low-charged nanofibrillated cellulose be produced by the addition of carboxymethyl cellulose? Nord Pulp Paper Res J 30(4):568-577. doi:10.3183/ NPPRJ-2015-30-04-p568-577

Naderi A, Erlandsson J, Sundström J, Lindström T (2016a) Enhancing the properties of carboxymethylated nanofibrillated cellulose by inclusion of water in the pretreatment process. Nord Pulp Paper Res J 31(3):372-378

Naderi A, Lindström T, Erlandsson J, Sundström J, Flodberg G (2016b) A comparative study of the properties of three nanofibrillated cellulose systems that have been produced at about the same energy consumption levels in the mechanical delamination step. Nord Pulp Paper Res J 31(3):364-371

Noguchi Y, Noishiki Y, Homma I (2014) Phosphoric acid-esterified cellulose fibers and production method thereof. WO2014185505A1

Siró I, Plackett D, Hedenqvist M, Ankerfors M, Lindström T (2011) Highly transparent films from carboxymethylated microfibrillated cellulose: the effect of multiple homogenization steps on key properties. J Appl Polym Sci 119(5):2652-2660. doi:10.1002/app.32831

Sjöstedt A, Wohlert J, Larsson PT, Wågberg L (2015) Structural changes during swelling of highly charged cellulose fibres. Cellulose 22(5):2943-2953. doi:10.1007/s10570-0150701-4

Tejado A, Alam MN, Antal M, Yang H, van de Ven TGM (2012) Energy requirements for the disintegration of cellulose fibers into cellulose nanofibers. Cellulose 19(3): 831-842. doi:10.1007/s10570-012-9694-4

Wågberg L, Winter L, Ödberg L, Lindström T (1987) On the charge stoichiometry upon adsorption of a cationic polyelectrolyte on cellulosic materials. Colloids Surf 27: 163-173

Wickholm K, Larsson PT, Iversen T (1998) Assignment of noncrystalline forms in cellulose I by CP/MAS ${ }^{13} \mathrm{C}$ NMR spectroscopy. Carbohyd Res 312(3):123-129. doi:10. 1016/S0008-6215(98)00236-5

Yano H, Hashimoto T, Sato A, Nakagaito AN, Kitagawa K, Semba T, Ito A (2011) Cationic microfibrillated plant nanofibers with good tensile strength, comprising plant fibers modified with quaternary ammonium group-containing compounds and manufacture thereof and composites therefrom. JP2011162608A 